\title{
The structure of a reduced form of OxyR from Neisseria meningitidis
}

\author{
Sarah Sainsbury', Jingshan Ren1', Joanne E Nettleship ${ }^{1}$, Nigel J Saunders², David I Stuart¹ and Raymond J Owens ${ }^{* 1}$
}

\begin{abstract}
Background: Survival of the human pathogen, Neisseria meningitidis, requires an effective response to oxidative stress resulting from the release of hydrogen peroxide by cells of the human immune system. In N. meningitidis, expression of catalase, which is responsible for detoxifying hydrogen peroxide, is controlled by OxyR, a redox responsive LysR-type regulator. OxyR responds directly to intracellular hydrogen peroxide through the reversible formation of a disulphide bond between C199 and C208 in the regulatory domain of the protein.

Results: We report the first crystal structure of the regulatory domain of an OxyR protein (NMB0173 from N. meningitidis) in the reduced state i.e. with cysteines at positions 199 and 208. The protein was crystallized under reducing conditions and the structure determined to a resolution of $2.4 \AA$. The overall fold of the Neisseria OxyR shows a high degree of similarity to the structure of a C199S mutant OxyR from E. coli, which cannot form the redox sensitive disulphide. In the neisserial structure, C199 is located at the start of helix a3, separated by $18 \AA$ from C208, which is positioned between helices a3 and a4. In common with other LysR-type regulators, full length OxyR proteins are known to assemble into tetramers. Modelling of the full length neisserial OxyR as a tetramer indicated that C199 and C208 are located close to the dimer-dimer interface in the assembled tetramer. The formation of the C199-C208 disulphide may thus affect the quaternary structure of the protein.

Conclusion: Given the high level of structural similarity between OxyR from N. meningitidis and E. coli, we conclude that the redox response mechanism is likely to be similar in both species, involving the reversible formation of a disulphide between C199-C208. Modelling suggests that disulphide formation would directly affect the interface between regulatory domains in an OxyR tetramer which in turn may lead to an alteration in the spacing/orientation of the DNA-binding domains and hence the interaction of OxyR with its DNA binding sites.
\end{abstract}

\section{Background}

The Neisseria genus, of Gram-negative Betaproteobacteria, contains two closely related human pathogens, $N$. meningitidis, a leading cause of bacterial meningitis and septicaemia and $N$. gonorrhoeae, that causes the sexually transmitted infection, gonorrhoea [1]. Survival of bacteria like Neisseria depends upon an effective response to oxidative challenges from the host environment, for example, reactive oxygen species (ROS), superoxide (. $\mathrm{O}^{2-}$ ), hydrogen peroxide $\left(\mathrm{H}_{2} \mathrm{O}_{2}\right)$ produced by phagocytes. To protect cellular components, including proteins and DNA, bacteria produce a range of enzymes, including

\footnotetext{
*Correspondence: ray@strubi.ox.ac.uk

1 The Oxford Protein Production Facility and Division of Structural Biology, Henry Wellcome Building for Genomic Medicine, University of Oxford, Roosevelt Drive, Oxford, OX3 7BN, UK

Full list of author information is available at the end of the article
}

catalases, peroxidases, oxidoreductases, and superoxide dismutases, whose expression is frequently controlled by redox-sensing transcription factors.

OxyR, widely known as a mediator of hydrogen peroxide-induced gene expression, is a redox-sensitive LysRtype transcription factor. Increased cellular hydrogen peroxide levels result in the oxidation of a pair of conserved cysteine residues present in the regulatory domain of OxyR [2] which leads in turn to the activation of transcription. In E. coli, OxyR regulates in excess of ten genes, including the hydrogen peroxide detoxifying enzyme catalase $(k a t G)$, glutathione reductase (gor $A)$, glutaredoxin 1 $(\operatorname{grx} A)$ and thioredoxin $2(\operatorname{trx} C)[3,4]$. The neisserial OxyR which shares $37 \%$ amino acid sequence identity with its $E$. coli orthologue appears to control expression of a smaller regulon comprising the genes encoding catalase (kat), glutathione oxidoreductase (gor) and peroxiredoxin oxi- 
doreductase (prx) [5]. The OxyR regulon appears to have an important role in pathogenesis since $N$. gonorrhoeae strains lacking either $o x y R$, prx or gor are deficient in both biofilm formation and show reduced survival in cervical epithelial models [5]. In fact, there are now several reports of OxyR being important for biofilm formation in other bacteria including Pseudomonas aeruginosa and Tannerella forsythia [6,7].

Crystal structures of the regulatory domains of wild type and a C199S mutant of E. coli OxyR have been reported. These structures, corresponding to oxidised and 'pseudo' reduced forms respectively, indicated that oxidation of OxyR leads to a local rearrangement of the C-terminal regulatory domain of the protein and formation of an intramolecular disulphide bridge between C199 and C208 [2]. Kim et al. [8] raised the possibility that the thiol group of the cysteine residues of OxyR may form other stable adducts in vivo including S-NO and SSG, through S-nitrosylation and S-glutathionylation respectively [8]. In contrast, Lee et al. [9] detected no such adducts and their experiments indicated that activation of OxyR via formation of an intramolecular disulphide follows a two-step mechanism. Initially, C199 is oxidised to a sulphenic acid that is relatively unstable and rapidly reacts with $\mathrm{C} 208$ to form an intramolecular bond [9].

Biochemical analyses have indicated that E. coli OxyR is tetrameric fitting the archetype for LysR regulators $[2,10]$ and is believed to interact with DNA as a dimer of dimers whose spacing/orientation are dependent upon the oxidation state of the protein [2]. Modelling of reduced and oxidised OxyR dimers indicates that the spacing of DNA binding domains in each dimer is unlikely to be altered by oxidation. This suggests that oxidation modulates DNA binding by modulating the dimer-dimer interaction [11].

In N. meningitidis, OxyR appears to both repress basal catalase activity and control enzyme induction in response to hydrogen peroxide. Thus, OxyR knock-out cells showed higher basal catalase levels than wild type cells and appeared less sensitive to hydrogen peroxide [12]. The single C199S $N$. meningitidis OxyR mutant strain behaved like the null mutant in both killing assays and primer extension analysis, in that it had neither the ability to repress or activate transcription of catalase. However, the C208A OxyR mutant strain showed an 'intermediate' phenotype; in killing assays, it was similar to wild type cells, suggesting that catalase production was repressed but in contrast to wild type cells, it was impaired in its response to hydrogen peroxide [12]. The physiological relevance of this is unclear, though it may indicate a functional role for a partially reduced form of the protein as proposed by leva et al [12].

As part of an investigation of the relationship between OxyR structure and its function in $N$. meningitidis, we have determined the crystal structure of the regulatory domain of the protein, in its authentically reduced form. The overall fold of the reduced neisserial OxyR shows a high degree of similarity to the structure of the C199S mutant OxyR (PDB code 1I69) from E. coli, which cannot form the redox sensitive disulphide.

By comparing the structure with those of the regulatory domains in three full length LysR structures, we have obtained some insight into how oxidation of the redox responsive disulphide may affect the quaternary structure of OxyR tetramers.

\section{Results \\ OxyR protein production and oxidation state analysis of the cysteine residues}

Selenomethionine labelled protein, corresponding to the regulatory domain of OxyR (RD-OxyR), was produced in E. coli for crystallization (Table 1). Crystal screening experiments were set up in both the presence and absence of the reducing agent Tris (2-carboxyethyl) phosphine (TCEP) (2 mM). Crystals only grew from the protein containing TCEP. Following this observation, we compared the oxidation state of the cysteine residues (C199, C208, and C305) in OxyR either treated with TCEP or untreated. The mass of the two protein samples was measured before and after addition of the alkylating agent, iodoacetamide (Figure 1). Prior to alkylation, the mass spectra of the untreated RD-OxyR shown in panel (a) contained two predominant peaks, with the main peak $(\mathrm{m} / \mathrm{z}$ 24726) fitting with the predicted mass (24722 Da) for selenomethionine labelled protein. The small, additional peak observed at $\mathrm{m} / \mathrm{z} 24747.5(+21.5 \mathrm{Da})$ corresponds to a sodium adduct of RD-OxyR. No peaks consistent with any other cysteine adducts were observed. After alkylation (panel b), the main peak occurred at $\mathrm{m} / \mathrm{z} 24783$, an increase of 57 Da compared to the control sample consistent with the addition of one acetamide group $\left(\mathrm{CH}_{2}-\mathrm{CO}-\mathrm{NH}_{2}\right)$ to one of the cysteine residues. A secondary peak was observed at $\mathrm{m} / \mathrm{z} 24899$, corresponding to the alkylation of all three cysteine residues. The alkylation of cysteine residues is dependent on the oxidation state, and accessibility, of the reactive thiol group. When the RD-OxyR was pre-treated with TCEP prior to alkylation two additional peaks were observed (panel c). The peak at $\mathrm{m} / \mathrm{z} 24842$ corresponded to alkylation of 2 cysteine residues and the peak at $\mathrm{m} / \mathrm{z} 24726$ corresponded to unmodified RD-OxyR. The presence of these two peaks suggests that the alkylation reaction had not gone to completion in this sample.

Overall, the results showed that the RD-OxyR protein produced in E. coli contained a mixed population of proteins with cysteine residues in both reduced and oxidised forms. Although the specific proportions of each species cannot be determined from the mass spectra obtained, 
Table 1: X-ray data collection and refinement statistics of OxyR (NMB0173)

\begin{tabular}{|c|c|c|}
\hline \multicolumn{3}{|l|}{ Data collection details: } \\
\hline X-ray source & ESRF BM14 & \\
\hline Data set & Peak & remote \\
\hline Wavelength $(\AA ̊)$ & 0.9785 & 0.9070 \\
\hline Space group & $P 21$ & \\
\hline Unit cell (Å) & $a=49.81, b=56.08, c=81.25, \beta=104.9^{\circ}$ & \\
\hline Resolution range $(\AA)$ & $30.0-2.40(2.49-2.40)$ & \\
\hline Unique reflections & $16661(1280)$ & $16617(1122)$ \\
\hline Completeness ${ }^{\mathrm{a}}(\%)$ & $96.8(76.1)$ & $95.1(65.4)$ \\
\hline Redundancy & $6.9(4.6)$ & $3.9(2.8)$ \\
\hline Average $I(/ \sigma l)$ & $18.4(2.6)$ & $12.7(2.0)$ \\
\hline $\mathrm{R}_{\text {merge }}$ & $0.129(0.554)$ & $0.113(0.547)$ \\
\hline \multicolumn{3}{|l|}{ Refinement statistics: } \\
\hline Resolution range $(\AA)$ & $30.0-2.40(2.49-2.40)$ & \\
\hline No. of reflections (working/test) & $32077 / 1617$ & \\
\hline$R$-factorb $\left(R_{\text {work }} / R_{\text {free }}\right)$ & $0.218 / 0.294$ & \\
\hline No. of atoms (protein/water) & $3254 / 98$ & \\
\hline Rms bond length deviation ( $\AA$ ) & 0.009 & \\
\hline Rms bond angle deviation $\left(^{\circ}\right)$ & 1.3 & \\
\hline Mean B-factor (protein/water[ $\left[\AA^{2}\right]$ ) & $55.7 / 54.3$ & \\
\hline \multicolumn{3}{|l|}{ Ramachandran plot: } \\
\hline Most favoured regions (\%) & 85.5 & \\
\hline Allowed regions (\%) & 15.5 & \\
\hline Disallowed regions(\%) & 0 & \\
\hline
\end{tabular}

due to potential differences in the ionization properties of the different redox forms, the results indicate that the OxyR protein was predominantly in the oxidised state with only one cysteine available for alkylation in the absence of reduction. Given that C305 is located at the Cterminus of the protein on the opposite face of the protein to C199 and C208 (Figure 1), it seems most likely that this is the un-paired cysteine. It follows that C199 and C208 are disulphide-linked in a significant proportion of the recombinant RD-OxyR, supporting the results for "air oxidised" E. coli OxyR, reported by Lee et al. [9] which contradicted earlier data of Kim et al. [8] who found no evidence of a C199-C208 disulphide.

\section{Description of the OxyR structure}

The $N$. meningitidis RD-OxyR structure was determined to a resolution of $2.4 \AA$ by multiple wavelength anomalous dispersion method using a selenomethionine-substituted protein. As expected from the requirement for
TCEP during crystallization, the protein was in the reduced form with no disulphide between C199 and C208. We observed no evidence for partial reduction of C199 in the electron density (Figure 2). The asymmetric unit contained a dimer arranged in the classical head-totail orientation, typical for regulatory domain structures of LysR transcription factors (Figure 3) which was first observed in the structure of the regulatory domain of $E$. coli CysB [13].

Each OxyR chain comprises two sub-domains, RD-I (residues 90-162 and 270-306) and RD-II (residues 163269) which contains the redox centre (C199 and C208). There are three residues missing from both the $\mathrm{N}$ - and $\mathrm{C}$ termini in the final model of the first monomer, (chain $\mathrm{A}$ residues 88-89 and 307). RD-I, which is more structurally conserved within the LysR family compared to RD-II, contains secondary structural elements of topology $\beta 1$ $\alpha 1-\beta 2-\alpha 3-\beta 3-t-\beta 4$ from the N-termini and $\beta 9-t-\alpha 6-\beta 10$ from the $\mathrm{C}$-termini. The $\beta 4$ and $\beta 9$ strands cross-over 


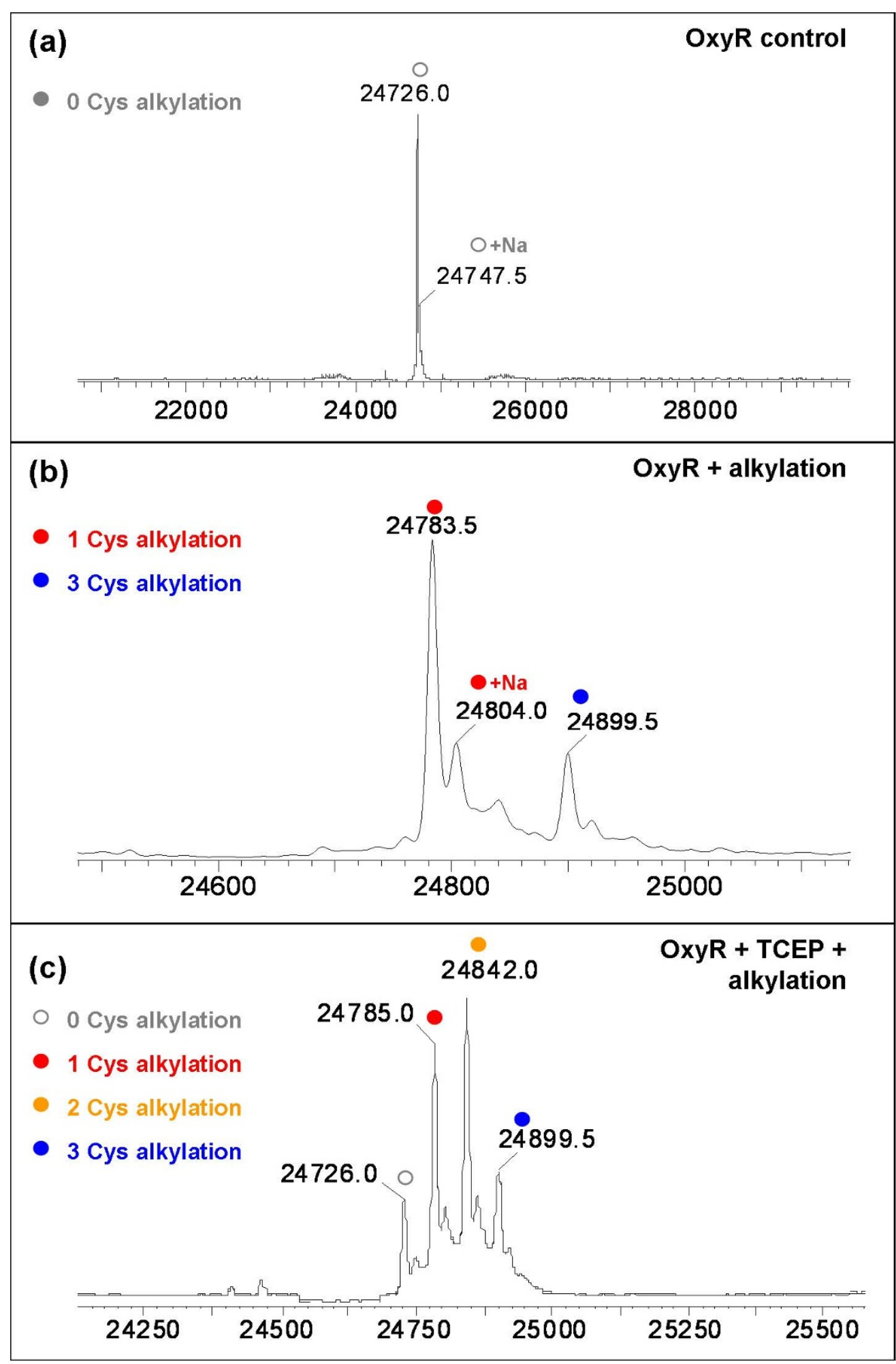

Figure 1 Alkylation and MS analysis of the oxidation state of the cysteine residues of the neisserial OxyR. Spectra: (a) OxyR control; (b) OxyR after alkylation with iodoacetamide; (c) OxyR after alkylation with iodoacetamide following prior reduction with TCEP. Minor peaks are sodium adducts of the corresponding, labelled major peaks. OxyR protein at $1 \mathrm{mg} / \mathrm{ml}$ in $20 \mathrm{mM}$ Tris pH 7.5, $200 \mathrm{mM} \mathrm{NaCl}$ was incubated with/without the addition of TCEP to $2 \mathrm{mM}$ at $37^{\circ} \mathrm{C}$ for $30 \mathrm{~min}$. An equal volume of alkylating buffer ( $20 \mathrm{mM}$ Tris pH 7.5, $0.1 \mathrm{mM}$ EDTA, $20 \mathrm{mM}$ iodoacetamide) was added and the samples were incubated for a further $1 \mathrm{~h}$. The samples were analysed using electrospray mass spectrometry using a Dionex Ultimate liquid chromatography system connected to a Waters Q-Tof Micro mass spectrometer [24]. 


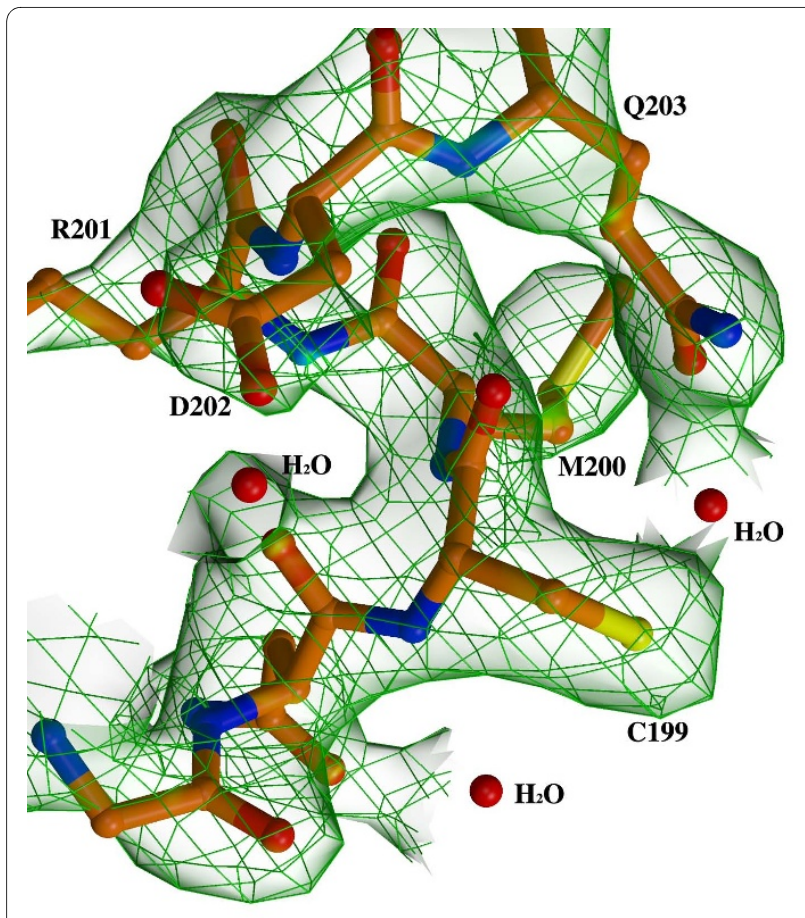

Figure $22 \mathrm{fo}-\mathrm{fc}$ map contoured at 1.0 sigma showing the electron density for part of alpha 3 helix that carrying the reduced residue Cys 199

between the two sub-domains. RD-II contains a central core of $4 \beta$ strands ( $\beta 5-\beta 8)$ surrounded by a series of $3^{10}$ $(\eta 1-\eta 2)$ and $\alpha$ helices $(\alpha 3-\alpha 5)$ (Figure 3$)$. The two redox sensitive cysteines (measured from the $\gamma \mathrm{S}$ of $\mathrm{C} 199$ and C208, chain A) are separated by $18.4 \AA$ and are located either end of helix $\alpha 3$ (C199-S207). Residues A212-Q218 form a second helix $(\alpha 4)$ which extends out of RD-II, at an angle of $\sim 75^{\circ}$ relative to $\alpha 3$ (Figure $4 \mathrm{a}$ ). Chain B is missing 20 residues in total (residues 88-89, 209-221 and 307) largely from RD-II. Disorder within this region of the regulatory domain was observed in both chains of the structure of the E. coli C199S OxyR mutant [2].

\section{Comparison of the E. coli and N. meningitidis OxyR regulatory domain structures}

The structure of reduced $N$. meningitidis OxyR (Chain A) was compared to that of the C199S OxyR mutant from $E$. coli (PDB code $1 \mathrm{I} 69$, chain B) which represents a 'pseudo reduced' form of the protein. Overall, the two structures proved to be very similar, superimposing with a root mean square deviation (RMSD) of $1.57 \AA$ for 193 equivalent $C \alpha$ atoms. In the overlay shown in Figure 4a, residue S199 of the 'pseudo reduced' E. coli structure and the corresponding C199 residue of neisserial OxyR are in almost exactly the same position. The side-chains of both residues are directed towards the interior of the protein, though C199 is more exposed to solvent, with a calcu- lated accessible surface area (ASA) of $32 \AA^{2}$ compared to $14 \AA^{2}$ for S199. Choi et al propose that in the reduced state, C199 in E. coli OxyR is stabilised by hydrophobic interactions involving L200, L224 and L224 and proximity to R266 which could form a charge interaction with an ionised C199 [2]. The corresponding residues (M200, I229, P246 and R270) are structurally conserved within neisserial OxyR, indicating that they are likely to have the same stabilising affect in this protein. By contrast, the side-chains of $\mathrm{C} 208$ are pointing in different directions in the two structures, with C208 buried in neisserial OxyR with an ASA of $5 \AA^{2}$ compared to $109 \AA^{2}$ for the surface exposed C208 in E. coli OxyR. Reactivity of C199 with peroxide is believed to initiate the redox switch in OxyR with oxidation to a sulphenic acid [9]. Formation of the disulphide with $\mathrm{C} 208$ requires significant local rearrangement of the polypeptide chain. In the structure of oxidised E. coli OxyR (PDB code 116A), the helix equivalent to the $\alpha 3$ helix in the neisserial structure (Figures 3 and 4 ) is unwound adopting a random coil type arrangement. Presumably, a similar conformational change would occur on oxidation of the $N$. meningitidis OxyR. Interestingly, residues that follow on from $\alpha 3$ helix in the reduced N. meningitidis OxyR structure (A212-Q218 in Chain A) form an alpha helix $(\alpha 4)$, whereas the equivalent residues in the E. coli C199S structure are disordered (PDB code $\underline{1169}$, chain $\mathrm{A}$, residues $210-215$ and chain $\mathrm{B}$, residues 211-216, are absent from the final model). In the N. meningitidis OxyR sequence there is an insertion following the $\alpha 4$ helix which is absent from the $E$. coli sequence and may account for the structural differences in this region (Figure 3). In the structure of oxidised E. coli OxyR, residues corresponding to helix $\alpha 4$ in the neisserial structure adopt a different secondary structure namely a short beta strand (residues, D214-D216) (Figure 4c). Whether helix $\alpha 4$ in neisserial OxyR would be preserved following formation of the C199-C208 disulphide is uncertain, though examination of the reduced $N$. meningitidis OxyR structure suggests that helix $\alpha 4$ could be re-positioned without loss of secondary structure.

\section{Modelling the full length OxyR}

Biophysical characterization of full-length E. coli OxyR indicates that it is tetrameric in solution $[10,14]$. Furthermore, a C199S mutant that models the reduced state of the protein also behaves predominantly as a tetramer according to size exclusion chromatography [10]. Thus, OxyR appears to be typical of LysR family regulators which are generally observed to form tetramers (e.g. Dntr [15], OccR [16] and CysB [17]). An exception to this is the $\mathrm{CrgA}$ regulator, also from $N$. meningitidis, which we have previously shown to be octameric in solution and in the crystal structure [18]. Cluster analysis of all known LysR sequences has shown that CrgA belongs to a sub-group 


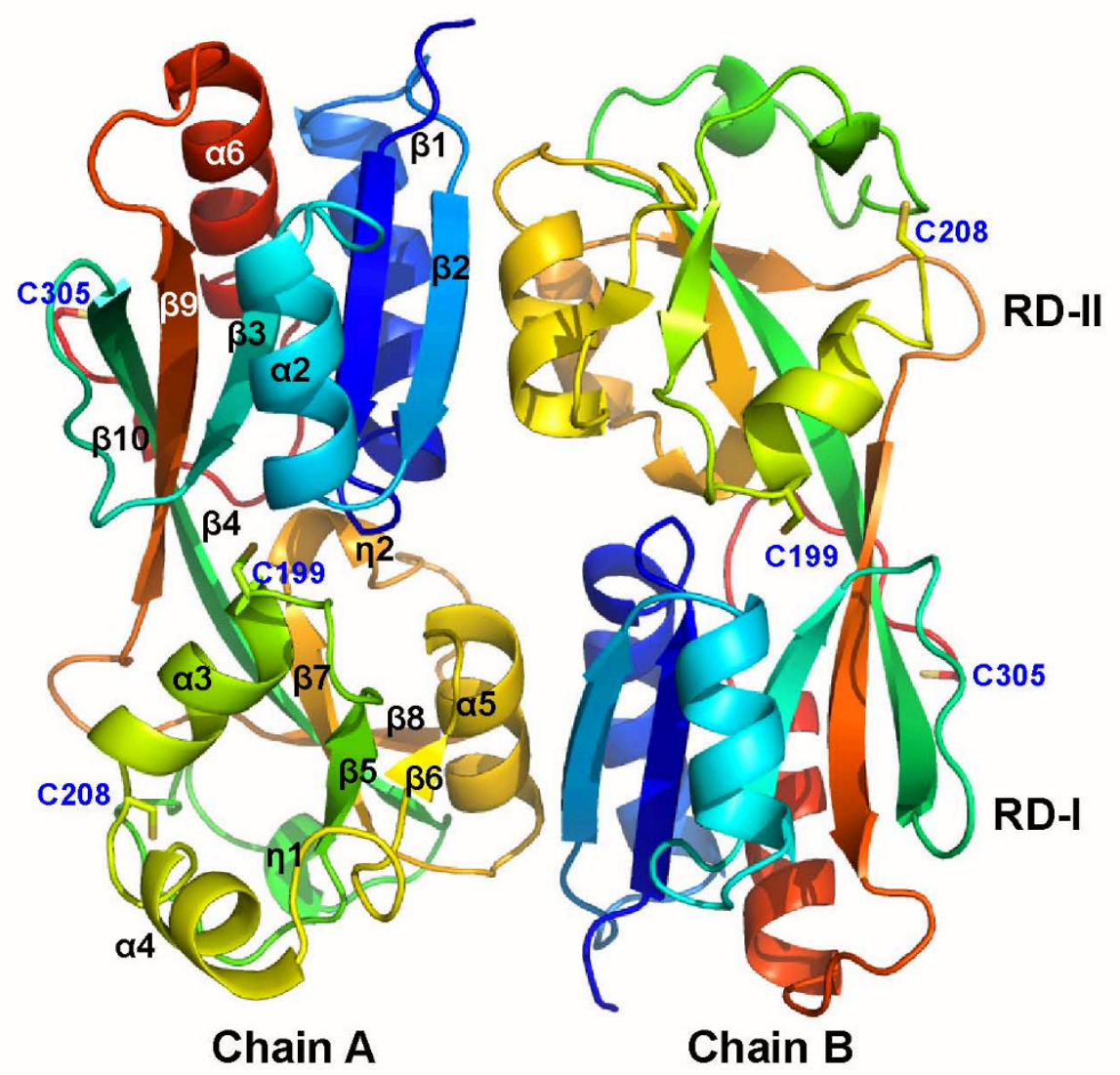

OxyR_MC58

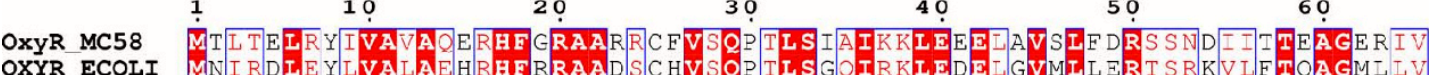
OXYR_ECOLI MNIRDLEYLVALAEHRHFRRAADSCHVSQP TLSGIRKLEDELGVMLLERTSRKVLFTOAGMLIV

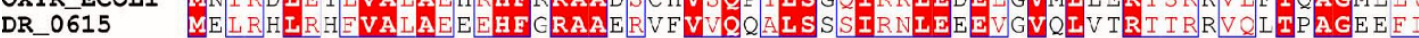

OxyR_MC58

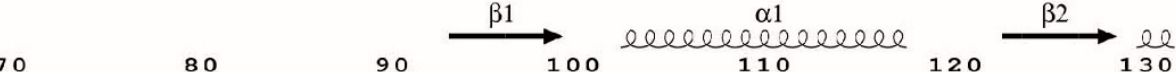

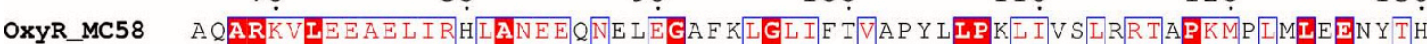

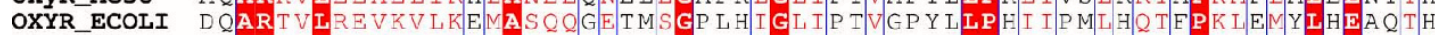

DR_0615 IGARATLAQAAQTVERARRAAGGEVGRLTVGEVSGLAF GGLPEIVRRERELYPAVSVIDLRELTAG

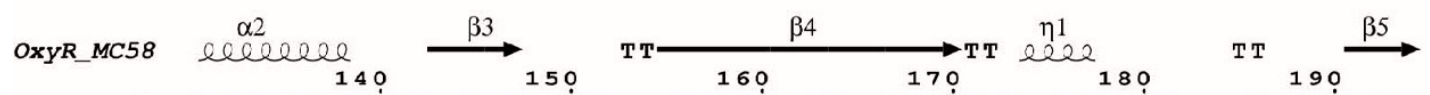

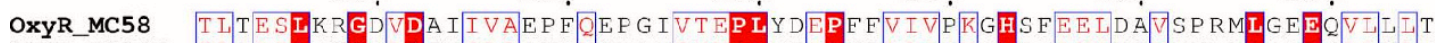
OXYR ECOLI QLLAOLD SGRLDCVI LALLKESEAF I E VPLE DEPMLLA IYEDHPWANRECVPMADLAGEKL LMLE

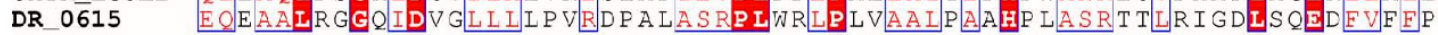

OxyR MC58

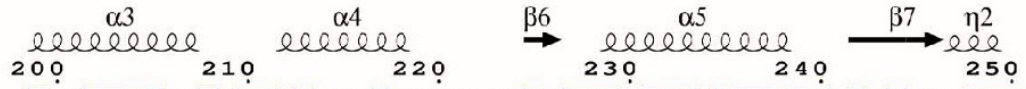

OxyR MC58 EGN . . CMRDQVLS SCSEIARKQRI Q L TNTLQGSSINTIRHMVASGLAISVILA TAIT. ANDHMI

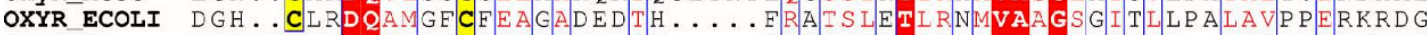

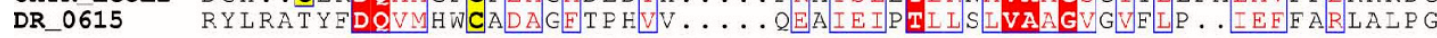

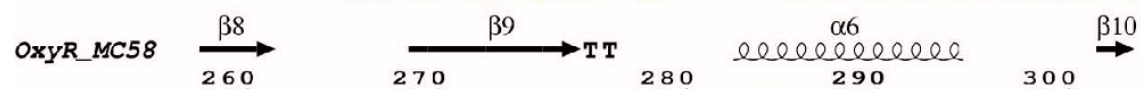

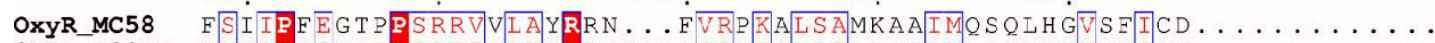
OXYR_ECOLI VVYLPCIKREPRRIIGIVYRPGSPLRSRYEQLAEAIRARMDGHFDKVLKQAV . . . . . . . . DR_0615 VVYRPLEG. APVIETVAVWRRAA.AGEQSTRPTVQAFLQVAERVLSGENGÄGEATPLPVLPTEPPR

Figure 3 Neisserial OxyR dimer solved to $2.4 \AA ̊$ resolution and multiple sequence alignment of OxyR homologues. Abbreviations: OXYR_ECOLI, OxyR of E. coli; OXYR_MC58, OxyR of N. meningitidis MC58; DR_0615, putative OxyR (gene dr0615) from D. radiodurans. The secondary structural elements of the neisserial OxyR are shown above the alignment. 


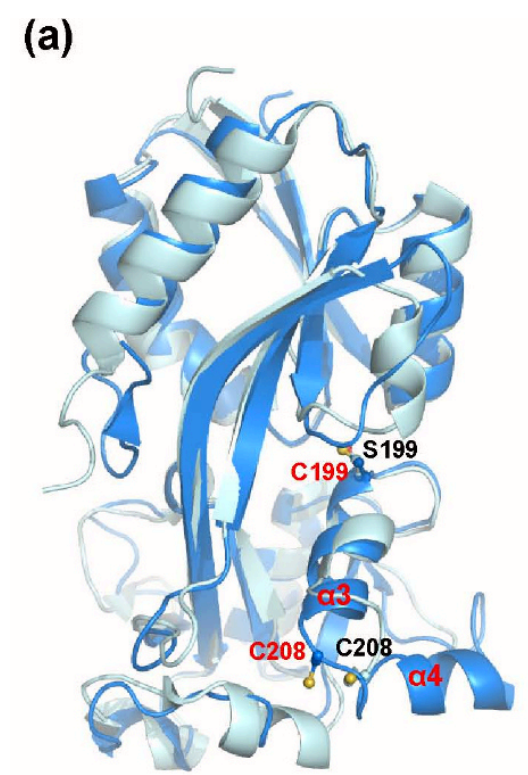

(b)

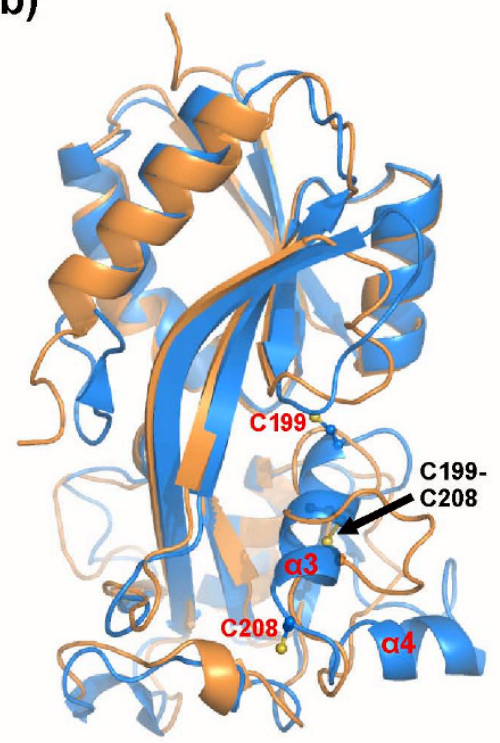

(c)

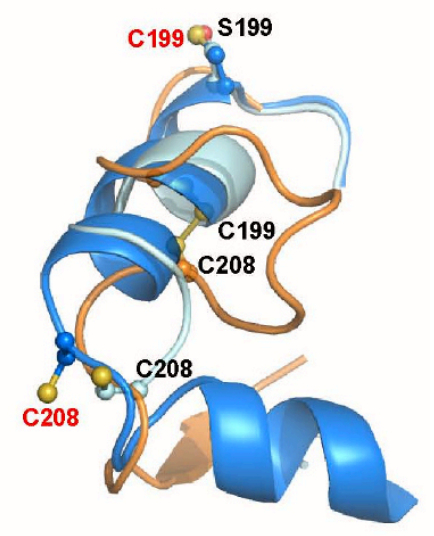

Figure 4 Structural comparison of the $N$. meningitidis OxyR and E. coli OxyR. (a) Superimposition of the N. meningitidis OxyR (blue) and the 'reduced' C199S E. coli OxyR (grey, PDB code 1169). (b) Superimposition of the N. meningitidis OxyR (blue) and the oxidised E. coli OxyR (orange, PDB code 116A). (c) Comparison of the redox active centre of, N. meningitidis OxyR (blue); 'reduced' C199S E. coli OxyR (grey); and oxidised E. coli OxyR (orange).

that is distinct from the majority of LysR including OxyR [18].

To visualise how the reduced form of neisserial RDOxyR might be arranged in the full length protein, we superimposed the structure onto that of three LysR tetramers whose crystal structures have been determined. These were CbnR from Ralstonia eutropha NH9 which regulates catechol metabolism ([19] PDB id 1IZ1) and two putative LysR transcription factors from Pseudomonas aeruginosa (PA0477, PDB id 2ESN and PA01, PDB id 3FZV unpublished data). Although each tetramer consists of a dimer of dimers, the arrangement of the dimers in each tetrameric assembly is significantly different. This in turn determines the relative positions of the N-terminal DNA binding domains. The OxyR regulatory domain superimposed well on each structure, with a RMSD of $\sim 2.0 \AA$ for 160 (2ESN), 171 (1IZ1) and 175 (3FZV) equivalent $\mathrm{C} \alpha$ atoms (Figure 5) and corresponding sequence identities of $15.6 \%$ (2ESN), 17.5\% (1IZ1) and $24.6 \%$ (3FZV) respectively From a visual inspection of the overlayed structures, it is possible to see how formation of the C199-208 on oxidation of OxyR could affect the quaternary arrangement of the protein. As described below, the situation is different for each form of tetrameric assembly represented by the three structures. In the CbnR structure, each dimer, formed between chains A \& $\mathrm{Q}$, or B \& P, contains one monomer in a compact form (chains $A$ and $P$ ) and one in an extended conformation (chains B and Q). The only contact between the regulatory domains of the two dimers is found in the core of the tetramer, at the interface formed primarily between the C-terminal $\alpha$ helices of the two RD-II sub-domains of chains A \& P (Figure 5a). The overlay with reduced OxyR shows that the helices, $\alpha 3$ and $\alpha 4$ which are located either side of $\mathrm{C} 208$ would contribute to the dimer-dimer interface in this model of OxyR (Figure 5a). Therefore, the formation of the C199-C208 disulphide bridge which involves a disruption of the $\alpha 3$ helix (Figure 4 and [2]) would be expected to alter the dimer-dimer interface and hence the quaternary structure of the OxyR tetramer. In the PA01 tetramer, the regulatory domains also contribute to the dimer-dimer interface, but in this case the domains of all four subunits are involved (Figure 5b). In a "head-to-tail" arrangement of the regulatory domains of the two dimers, the $\alpha 3$ and $\alpha 4$ helices of the overlayed reduced OxyR still form part of the dimer-dimer interface (A:C and B:D, Figure $5 \mathrm{~b}$ ) packing onto residues H153I159 (residues in PA01) in RDI of the adjacent dimer. Again, formation of the disulphide would be anticipated to alter the dimer-dimer packing. The PA0477 presents a very different tetrameric arrangement compared to the other two LysR structures in that there is no contact between the regulatory domains of the two dimers (A:C and $\mathrm{B}: \mathrm{D}$ Figure $5 \mathrm{c}$ ) rather the dimer-dimer interfaces are formed by the DNA binding domains and interdomain linker helices. In this case, superimposition of the reduced OxyR regulatory domain indicates that the $\alpha 3$ and $\alpha 4$ helices contribute to the interface between adjacent subunit monomers packing onto the second helix of the DNA binding domain (A and C, B and D Figure 5c). 
(a)

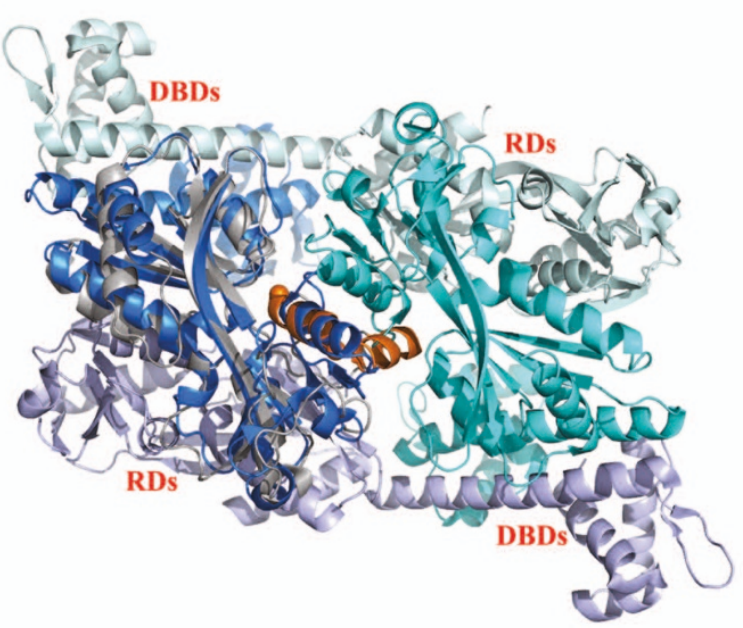

(b)

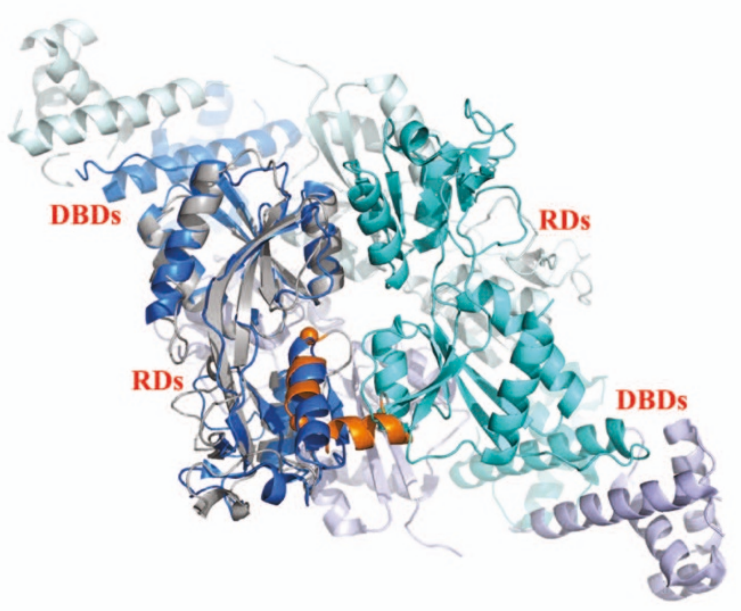

(c)

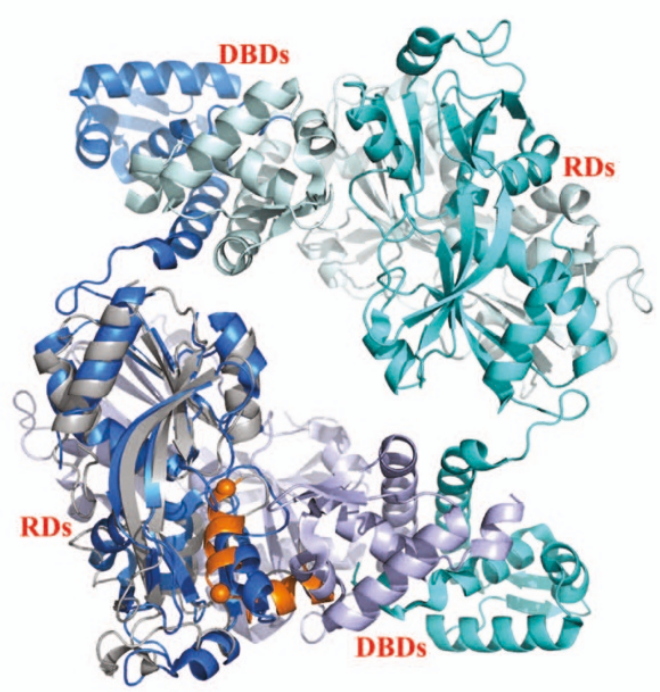

Figure 5 Modelling of a neisserial OxyR tetramer. The OxyR regulatory domain shown in grey was superimposed onto the tetrameric structures of (a) CbnR (PDB code 1IZ1). (b) PA01 (PDB id $\underline{3 F Z V}$ ) and (c) PA0477 (PDB id $\underline{2 E S N}$ ). The a3 and a4 helices which flank the C199 and C208 residues (shown as solid spheres) are coloured orange. The two pairs of dimers which form each tetramer are coloured bright/light blue (A:C in PA01 and PA0477; A:Q in CbnR) and bright/light cyan (B:D in PA01 and PA0477; B:P in CbnR). 
In the absence of a full length OxyR structure we do not know which (if any) of the three structures we have considered provides the best model of the OxyR tetramer. It may well be that OxyR can adopt all the configurations represented by the three structures. However in two of the three possible models (Figure $5 \mathrm{a}$ and $5 \mathrm{~b}$ ) the redox sensitive disulphide is located at a dimer-dimer interface. This provides a plausible explanation of how formation of the disulphide in response to oxidative challenge, may modulate the arrangement of subunits in the tetrameric assembly. This in turn would affect the relative positions of the DNA binding domains and hence account for the changes in the interaction with target promoters that has been observed experimentally. The oxidation of C199C208 would have a more direct effect on the position or orientation of the DNA binding domains if OxyR adopted the tetrameric configuration of PA0477 (Figure 5c).

\section{Discussion}

As reviewed by Paget and Buttner in 2003 [20] two different mechanisms for OxyR regulation have been proposed by the Stamler and Storz research groups respectively. The Storz group suggest that OxyR functions as an on-off switch, converting between oxidised, with an intramolecular disulphide, and reduced forms; whereas the Stamler group, who detected no disulphide bond, suggest that OxyR has a graded response with $\mathrm{C} 199$ being subject to different modifications (e.g. S-OH, SNO or S-SG) producing different transcriptional outcomes [20]. Consistent with the results of Storz et al., our mass spectrometry data indicated that the nessierial OxyR contains a disulphide presumably the result of air oxidation during purification; no cysteine adducts were detected. This favours an activation mechanism based on the reversible oxidation of the conserved cysteine pair (residues 199 and 208). Molecular modelling with the reduced and oxidised OxyR structures indicated that formation of such an intramolecular disulphide bond would affect the dimer-dimer interface of the neisserial OxyR tetramer. A small movement at this pivot point, in the centre of the tetramer, could potentially translate into a much larger movement of the heads of the DNA binding domains (Figure 5). An indication that oxidation of OxyR is associated with a change in DNA binding comes from studies of E. coli OxyR. Wild type and C199S OxyR mutant were compared in gel shift and foot printing experiments and shown to differ in their DNA binding properties [11]. In the case of its own promoter, the results showed that the oxidised form of the protein contacted the oxyR promoter at four consecutive major grooves consistent with a tetrameric assembly. By contrast the C199S mutant protected a more extended region containing two binding sites, each comprising two major grooves separated by one helical turn. This indicates that the oxidised form of E. coli OxyR adopts a more compact structure, with the DNA binding pairs closer together, compared to the reduced form [14]. Although the $N$. meningiditis $\mathrm{OxyR}$ structure provides some clues as to how oxidation of OxyR could alter its interaction with DNA, it does not readily explain the phenotypic differences between the single C199S and C208A mutant neisserial strains reported by Ieva et al. [12]. The C199S mutant does not appear to be functionally active (i.e. it cannot repress or activate expression) whereas the C208A is able to repress but not activate catalase expression. Formation of the C199-C208 disulphide bond is presumably required for activation of the kat promoter since this is blocked by mutation of either cysteine residue. However, repression of the kat promoter only requires C199 since the C208A mutant retains repressive activity whereas the C199S is inactive. To ascertain whether this reflects conformational differences between the two proteins will require further structural studies and comparison with the authentically reduced protein reported here. Intriguingly, an OxyR homologue from Deinococcus radiodurans has recently been reported that contains only one of the conserved cysteines (C210), corresponding to the $\mathrm{C} 208$ of E. coli and N. meningitidis OxyR (Figure 2) [21]. Deinococcus strains in which the oxyR gene had been either deleted or substituted with a C210A mutant gene, showed increased sensitivity to hydrogen peroxide challenge and no increase in catalase activity compared to wild type bacteria. C210 residue was also shown to be oxidised to a sulphenic acid in vitro under conditions of oxidative stress [21]. It would be very interesting to compare the structure of the Deinococcus OxyR with those of $N$. meningitidis and $E$. coli in order to understand better the role of the individual cysteines in the functional responses of these proteins.

\section{Conclusions}

The structure of the regulatory domain of $N$. meningitidis OxyR represents the first determined for a wildtype OxyR protein in its reduced form. Although there are subtle differences, overall, the structures of neisserial and E. coli OxyR are very similar and hence the mechanism of activation of these regulators is likely to be similar in both species. Modelling of an N. meningitidis OxyR tetramer suggested that the redox active cysteines, C199 and C208, are located at the interface of the two dimers that form the LysR tetramer. This location suggests that formation of the C199-C208 disulphide would affect the relative disposition of the two dimers within the tetramer which in turn may alter the spacing/orientation of the DNA binding domains and hence the interaction of OxyR with its DNA binding sites. 


\section{Methods}

\section{Protein production}

The oxyR gene (NMB0173) was amplified from genomic DNA using the forward primer 5'-AAGTTCTGTTTCAGGG CCCGGAGCTGGAGGGTGCGTTCAAAC-3' and reverse primer 5'-ATGGTCTAGA AAGCTTTACTAGTCGCAGATAAAAC TTACCCCG$3^{\prime}$ and cloned into pOPINF using In-Fusion ${ }^{\text {тм }}$ cloning technology as reported [22]. Selenomethionine labelled protein was produced from the pOPINF OxyR expression construct containing OxyR residues 88-307 and an N-terminal cleavable His tag [MAHHHHHHSSGLEVLFQ GP, ( human rhinovirus $3 \mathrm{C}$ protease cleavage site)] in $E$. coli strain B834 using SelenoMet media (Molecular dimensions). The recombinant protein was purified using Nickel affinity chromatography and subsequently size exclusion chromatography following methods previously described [23]. 100\% selenium incorporation was confirmed by mass spectrometry [24].

\section{Crystallography and structure solution}

After removal of the N-terminal His tag, the selenomethionine labelled OxyR protein was concentrated to $9 \mathrm{mg} / \mathrm{ml}$ in $20 \mathrm{mM}$ Tris $\mathrm{pH} 7.5,200 \mathrm{mM} \mathrm{NaCl}$. Fractions were pooled and concentrated in Vivaspin 15 concentrators (Vivascience). Crystallization screening experiments in nanodrops were performed as described [25]. Following unsuccessful initial crystallization screening experiments, further crystal trials were set up with the same batch of protein, but with $2 \mathrm{mM}$ of the reducing agent, TCEP, present. Under these conditions the protein crystallized and the crystals were subsequently optimized using the standard three row optimization procedure detailed by Walter et al. [26]. The crystal used for data collection grew in a drop of $200 \mathrm{nl}$ protein and $100 \mathrm{nl}$ of reservoir solution containing 20\% w/v PEG 3350, $0.2 \mathrm{M}$ calcium acetate (Hampton PEG/Ion Screen, condition 28). The crystal was flash frozen in a cryoprotectant solution of $25 \% \mathrm{v} / \mathrm{v}$ ethylene glycol and $75 \%$ of the reservoir solution and maintained at $100 \mathrm{~K}$ under a stream of nitrogen gas during data collection. Multiple wavelength anomalous dispersion data were collected at two wavelengths at beamline BM14, the ESRF (Grenoble, France) to $2.4 \AA$ resolution. The HKL2000 suite was used to integrate and scale the data [27]. The SHELX program suite was used to monitor the anomalous signal and to identify the selenium sites [28]. SOLVE/RESOLVE [29] were then used for refinement of selenium positions and phase improvement, combined with automated model building. Refinement was carried out with CNS [30] using simulated annealing and positional refinement with main chain NCS restraints, followed by individual isotropic B factor refinement. The final stages of refinement were carried out with PHENIX using anomalous scattering factors
[31]. The coordinates and structure factors have been deposited in the Protein Data Bank under accession number 3JV9.

\section{Bioinformatics and structure analysis}

Structural alignments were carried out using the programme SHP [32]. The figures were produced using Pymol [33]. Sequences were aligned using ClustalW [34] and displayed with secondary structures using ESPript2.2 [35].

\section{MS analysis of the oxidation state of cysteine residues}

OxyR protein at $1 \mathrm{mg} / \mathrm{ml}$ in $20 \mathrm{mM}$ Tris $\mathrm{pH} 7.5,200 \mathrm{mM}$ $\mathrm{NaCl}$ was incubated with/without the addition of TCEP to $2 \mathrm{mM}$ at $37^{\circ} \mathrm{C}$ for $30 \mathrm{~min}$. An equal volume of alkylating buffer (20 mM Tris pH 7.5, $0.1 \mathrm{mM}$ EDTA, $20 \mathrm{mM}$ iodoacetamide) was added and the samples were incubated for a further $1 \mathrm{~h}$. The samples were analysed using electrospray mass spectrometry using a Dionex Ultimate liquid chromatography system connected to a Waters QTof Micro mass spectrometer [24].

\section{Authors' contributions}

NJS, DIS and RJO initiated the study. SS cloned, purified and crystallized the protein, JN carried out analysis by mass spectrometry, JR and SS collected and processed the diffraction data, modelled and refined the structure. SS, JR and $\mathrm{RJO}$ analysed the structure and wrote the paper which was read and approved by all authors.

\section{Acknowledgements}

This work was supported by UK Medical Research Council and the Biotechnology Biological Research Council. S.S. was funded by a MRC Research Studentship. We would like to thank the beam line staff at ESRF BM14 and ID14.4 for assistance with data collection.

\section{Author Details}

'The Oxford Protein Production Facility and Division of Structural Biology, Henry Wellcome Building for Genomic Medicine, University of Oxford, Roosevelt Drive, Oxford, OX3 7BN, UK and ${ }^{2}$ The Bacterial Pathogenesis and Functional Genomics Group, The Sir William Dunn School of Pathology, University of Oxford, South Parks Road, Oxford, OX1 3RE. UK

Received: 4 November 2009 Accepted: 17 May 2010

Published: 17 May 2010

\section{References}

1. Rosenstein NE, Perkins BA, Stephens DS, Popovic T, Hughes JM: Meningococcal disease. NEngl J Med 2001, 344:1378-1388.

2. Choi H, Kim S, Mukhopadhyay P, Cho S, Woo J, Storz G, Ryu S: Structural basis of the redox switch in the OxyR transcription factor. Cell 2001, 105:103-113.

3. Christman MF, Storz G, Ames BN: OxyR, a positive regulator of hydrogen peroxide-inducible genes in Escherichia coli and Salmonella typhimurium, is homologous to a family of bacterial regulatory proteins. Proc Natl Acad Sci USA 1989, 86:3484-3488.

4. Zheng M, Wang X, Templeton LJ, Smulski DR, LaRossa RA, Storz G: DNA microarray-mediated transcriptional profiling of the Escherichia coli response to hydrogen peroxide. J Bacterio/ 2001, 183:4562-4570.

5. Seib KL, Wu HJ, Srikhanta YN, Edwards JL, Falsetta ML, Hamilton AJ Maguire TL, Grimmond SM, Apicella MA, McEwan AG, et al:: Characterization of the OxyR regulon of Neisseria gonorrhoeae. Mol Microbiol 2007, 63:54-68

6. Panmanee W, Hassett DJ: Differential roles of OxyR-controlled antioxidant enzymes alkyl hydroperoxide reductase (AhpCF) and catalase (KatB) in the protection of Pseudomonas aeruginosa against 
hydrogen peroxide in biofilm vs. planktonic culture. FEMS microbiology letters 2009, 295:238-244.

7. Honma K, Mishima E, Inagaki S, Sharma A: The OxyR homologue in Tannerella forsythia regulates expression of oxidative stress responses and biofilm formation. Microbiology 2009, 155:1912-1922.

8. Kim SO, Merchant K, Nudelman R, Beyer WF Jr, Keng T, DeAngelo J, Hausladen A, Stamler JS: OxyR: a molecular code for redox-related signaling. Cell 2002, 109:383-396.

9. Lee C, Lee SM, Mukhopadhyay P, Kim SJ, Lee SC, Ahn WS, Yu MH, Storz G, Ryu SE: Redox regulation of OxyR requires specific disulfide bond formation involving a rapid kinetic reaction path. Nat Struct Mol Biol 2004, 11:1179-1185.

10. Kullik I, Stevens J, Toledano MB, Storz G: Mutational analysis of the redoxsensitive transcriptional regulator OxyR: regions important for DNA binding and multimerization. J Bacteriol 1995, 177:1285-1291.

11. Zaim J, Kierzek AM: The structure of full-length LysR-type transcriptional regulators. Modeling of the full-length OxyR transcription factor dimer. Nucleic Acids Res 2003, 31:1444-1454

12. leva R, Roncarati D, Metruccio MM, Seib KL, Scarlato V, Delany I: OxyR tightly regulates catalase expression in Neisseria meningitidis through both repression and activation mechanisms. Mol Microbio/ 2008, 70:1152-1165.

13. Tyrrell R, Verschueren KH, Dodson EJ, Murshudov GN, Addy C, Wilkinson $\mathrm{AJ}$ : The structure of the cofactor-binding fragment of the LysR family member, CysB: a familiar fold with a surprising subunit arrangement. Structure 1997, 5:1017-1032

14. Toledano MB, Kullik I, Trinh F, Baird PT, Schneider TD, Storz G: Redoxdependent shift of OxyR-DNA contacts along an extended DNAbinding site: a mechanism for differential promoter selection. Cell 1994, 78:897-909.

15. Smirnova IA, Dian C, Leonard GA, McSweeney S, Birse D, Brzezinski P. Development of a bacterial biosensor for nitrotoluenes: the crystal structure of the transcriptional regulator DntR. J Mol Biol 2004, 340:405-418.

16. Akakura R, Winans SC: Constitutive mutations of the OccR regulatory protein affect DNA bending in response to metabolites released from plant tumors. J Biol Chem 2002, 277:5866-5874.

17. Hryniewicz MM, Kredich NM: Stoichiometry of binding of CysB to the cys $\mathrm{JH}$, cysK, and cysP promoter regions of Salmonella typhimurium. J Bacteriol 1994, 176:3673-3682

18. Sainsbury S, Lane LA, Ren J, Gilbert RJ, Saunders NJ, Robinson CV, Stuart $\mathrm{Dl}$, Owens RJ: The structure of CrgA from Neisseria meningitidis reveals a new octameric assembly state for LysR transcriptional regulators. Nucleic Acids Res 2009

19. Muraoka S, Okumura R, Ogawa N, Nonaka T, Miyashita K, Senda T: Crystal structure of a full-length LysR-type transcriptional regulator, CbnR: unusual combination of two subunit forms and molecular bases for causing and changing DNA bend. J Mol Biol 2003, 328:555-566.

20. Paget MS, Buttner MJ: Thiol-based regulatory switches. Annu Rev Genet 2003, 37:91-121.

21. Chen H, Xu G, Zhao Y, Tian B, Lu H, Yu X, Xu Z, Ying N, Hu S, Hua Y: A novel OxyR sensor and regulator of hydrogen peroxide stress with one cysteine residue in Deinococcus radiodurans. PLOS ONE 2008, 3:e1602

22. Berrow NS, Alderton D, Sainsbury S, Nettleship J, Assenberg R, Rahman N, Stuart DI, Owens RJ: A versatile ligation-independent cloning method suitable for high-throughput expression screening applications. Nucleic Acids Res 2007, 35:e45.

23. Sainsbury S, Ren J, Saunders NJ, Stuart DI, Owens RJ: Crystallization and preliminary X-ray analysis of CrgA, a LysR-type transcriptional regulator from pathogenic Neisseria meningitidis MC58. Acta Crystallogr Sect $F$ Struct Biol Cryst Commun 2008, 64:797-801.

24. Nettleship JE, Brown J, Groves MR, Geerlof A: Methods for protein characterization by mass spectrometry, thermal shift (ThermoFluor) assay, and multiangle or static light scattering. In Structural Proteomics: High-throughput Methods Volume 426. Edited by: Kobe B, Guss M, Huber T. Humana Press; 2008:299-318.

25. Walter TS, Diprose J, Brown J, Pickford M, Owens RJ, Stuart DI, Harlos K: A procedure for setting up high-throughput nanolitre crystallization experiments. I. Protocol design and validation. Journal of Applied Crystallography 2003, 36:308-314.

26. Walter TS, Diprose JM, Mayo CJ, Siebold C, Pickford MG, Carter L, Sutton GC, Berrow NS, Brown J, Berry IM, et al:: A procedure for setting up high- throughput nanolitre crystallization experiments. Crystallization workflow for initial screening, automated storage, imaging and optimization. Acta Crystallogr D Biol Crystallogr 2005, 61:651-657.

27. Otinowski Z, Minor W: Processing of X-ray diffraction data collected in oscillation mode. Methods Enzymology 1997, 276:307-326.

28. Sheldrick GM: A short history of SHELX. Acta Crystallogr A 2008 , 64:112-122.

29. Terwilliger T: SOLVE and RESOLVE: automated structure solution, density modification and model building. I Synchrotron Radiat 2004, 11:49-52.

30. Brunger AT, Adams PD, Clore GM, DeLano WL, Gros P, Grosse-Kunstleve RW, Jiang JS, Kuszewski J, Nilges M, Pannu NS, et al.: Crystallography \& NMR system: A new software suite for macromolecular structure determination. Acta crystallographica 1998, 54:905-921.

31. Zwart PH, Afonine PV, Grosse-Kunstleve RW, Hung LW, loerger TR, McCoy AJ, McKee E, Moriarty NW, Read RJ, Sacchettini JC, et al: Automated structure solution with the PHENIX suite. Methods Mol Biol 2008, 426:419-435.

32. Stuart DI, Levine M, Muirhead H, Stammers DK: Crystal structure of cat muscle pyruvate kinase at a resolution of 2.6 A. J Mol Biol 1979, 134:109-142.

33. The PyMOL Molecular Graphics System [http://www.pymol.org]

34. Chenna R, Sugawara H, Koike T, Lopez R, Gibson TJ, Higgins DG, Thompson JD: Multiple sequence alignment with the Clustal series of programs. Nucleic acids research 2003, 31:3497-3500

35. Gouet P, Courcelle E, Stuart DI, Metoz F: ESPript: analysis of multiple sequence alignments in PostScript. Bioinformatics 1999, 15:305-308.

doi: $10.1186 / 1472-6807-10-10$

Cite this article as: Sainsbury et al., The structure of a reduced form of OxyR from Neisseria meningitidis BMC Structural Biology 2010, 10:10

\section{Submit your next manuscript to BioMed Central and take full advantage of:}

- Convenient online submission

- Thorough peer review

- No space constraints or color figure charges

- Immediate publication on acceptance

- Inclusion in PubMed, CAS, Scopus and Google Scholar

- Research which is freely available for redistribution 